\title{
NONUNIFORM DONNAN EQUILIBRIUM WITHIN BACTERIOPHAGES PACKED WITH DNA
}

\author{
Theo Odijk* \\ Section Theory of Complex Fluids \\ Faculty of Applied Sciences Delft \\ University of Technology Delft, the Netherlands \\ Flodder Slok \\ Department of Zoology \\ University of Amsterdam, the Netherlands
}

August 12, 2018

\begin{abstract}
The curvature stress of DNA packed inside a phage is balanced against its electrostatic self-interaction. The DNA density is supposed nonuniform and as a result the Donnan effect is also inhomogeneous. The coarse-grained DNA density is a nonlinear function of the DNA radius of curvature at a given position inside the bacteriophage. It turns out that a region (or regions) exists totally free from DNA. The size of such holes is computed.
\end{abstract}

* Address for correspondence :

T. Odijk, P.O. Box 11036, 2301 EA Leiden, the Netherlands

E-mail: odijktcf@wanadoo.nl 
The packing of DNA in cells and viruses are problems in the nanothermodynamics [1] of polyelectrolytes i.e. the systems are small and hence the free energies need not be extensive. A case in point is the compaction of DNA inside bacteriophages where the extensive self-energy of the DNA spool is balanced against the non-extensive energy of curvature arising mainly from a region of tightly wound DNA surrounding a hole [2]. The degree of non- extensivity depends on the problem at hand. For fairly large DNA globules that are compacted in solution, the shape is determined by non-extensive terms whereas the volume is not [3, 4.

In previous work [2, 3, 4] we assumed the DNA density in a coarse-grained continuum approximation to be homogeneous, but we relax this supposition here. This introduces a peculiar effect : the Donnan effect is now also nonuniform and it happens to compete with the curvature stress which increases sharply in tightly wound regions. Although the uniform approximation of [2] agrees well with the DNA spacings within bacteriophage T7 determined experimentally [ [5], the forces encountered in single molecule experiments [ of DNA being forced into Phi 29 phage by the connector motor seem to overestimate the predicted forces [2, 7]. Thus a reinvestigation of at least one assumption is warranted.

Close-packed DNA in vitro has often been found to be a hexagonal liquid crystal 8, 9]. Sometimes, for long polydisperse DNA, the hexagonal phase appears to be precluded in favor of a possible "hexatic" phase [10] but a full investigation of the algebraic decay of the ovientational order has yet to be carried out. Here, for the sake of definiteness, we assume the DNA lattice inside a phage is hexagonal (for general, biologically oriented reviews on DNA packaging in bacteriophages, [11, 12]). However, such a lattice is probably rarely ever ideal but is bound to contain DNA crossovers leading to knotted entanglements upon release of the DNA from the phage [13, 14].

Theories of slightly deformed hexagonal phases have been set up previously [15, 16]. They apply to macroscopic or extensive systems. Here, our compacted DNA is of mesoscopic dimensions and its properties are dominated by nonextensive curvature stresses so we simply disregard fluctuations in the director $n(\mathbf{r})$ and coarse-grained DNA density $\rho(\mathbf{r})$ at position $\mathbf{r}$. The linear double-stranded DNA has only two ends so to a good approximation a well-known continuity law applies [17, 18.

$$
\nabla \cdot \rho \mathbf{n}=0
$$


We next assume absence of splay $(\nabla \cdot \mathbf{n}=0)$ since the splay elastic constant is expected to be very large. In general, at constant density, we would deal with the theory of so-called developable domains in describing the deformed hexagonal liquid crystal [19]. We here consider deformations of nonuniform densities in such a way that the director remains perpendicular to the density gradient. Under these conditions eq.(11) is obeyed.

Now the simplest total free energy of the DNA coil as a functional of the density $\rho(\mathbf{r})$ seems to be

$$
F_{\text {tot }}=F_{\text {int }}[\rho(\mathbf{r})]+U_{b}[\rho(\mathbf{r})]
$$

where $F_{\text {int }}$ is the free energy of the DNA interacting with itself in a hypothetically straightened hexagonal lattice (though it is inhomogeneous across the plane perpendicular to its central axis) and $U_{b}$ is the bending energy given by

$$
U_{b}=\frac{1}{2} P k_{B} T \int_{0}^{L} d s R_{c}^{-2}(s)
$$

Here, $P$ is the DNA persistence length, $k_{B}$ is Boltzmann's constant, $T$ is the temperature and $R_{c}$ is the radius of curvature of the DNA wormlike coil at position $s$ on the DNA contour of total length $L$. As already stated, undulations of the DNA will be disregarded in the tightly packed state we focus on. If we define the surface area $S(\mathbf{r})$ of the unit cell of the deformed hexagonal lattice at position $\mathbf{r}$, we may introduce a continuum approximation $S(\mathbf{r}) d s=d \mathbf{r}$ and $\rho(\mathbf{r})=1 / A S(\mathbf{r})$. The length of a DNA nonomer is $A$ so that $\rho(\mathbf{r})$ is a monomer density from now on.

$$
U_{b}=\frac{1}{2} P A k_{B} T \int_{V} d \mathbf{r} \frac{\rho(\mathbf{r})}{R_{c}^{2}(\mathbf{r})}
$$

The number of monomers inside the phage of volume $V$ is kept fixed.

$$
N=\frac{L}{A}=\int_{V} d \mathbf{r} \rho(\mathbf{r})
$$

Supposing gradients of $\rho(\mathbf{r})$ may be disregarded in $F_{\text {int }}$, we now minimize the total free energy with respect to $\rho(\mathbf{r})$ while accounting for the constraint eq.(5).

$$
\mu(\mathbf{r})+\frac{P A k_{B} T}{2 R_{c}^{2}(\mathbf{r})}+\text { const. }=0
$$

We have introduced the inhomogeneous bulk chemical potential of the DNA monomers $\left(\mu=\delta F_{\text {int }} / \delta \rho(\mathbf{r})\right)$. It is readily seen that the curvature term becomes appreciable for tightly wound regions appropriate for DNA packed in phages. 
Next, we need an expression for the DNA chemical potential. The phage is in thermodynamic equilibrium with a large reservoir containing a univalent salt of concentration $c_{s}$.

The phage is presumed to be free from multivalent ions. We first focus on a homogeneous hexagonal lattice of DNA and its Donnan equilibrium with such a reservoir. One convenient approximation for the osmotic pressure is due to Oosawa [20, 21]. For our purposes, we set the osmotic coefficient equal to a constant so we write the osmotic pressure $\pi_{o s}$ of the uniform bulk hexagonal phase with respect to the buffer as

$$
\begin{gathered}
\pi_{o s}=\left(\frac{A \rho_{0} k_{B} T}{2 Q}\right) h(w) \\
=\left(\frac{k_{B} T}{3^{\frac{1}{2}} H_{0}^{2} Q}\right) h(w) \\
w \equiv 2.3^{\frac{1}{2}} H_{0}^{2} Q c_{s}=4 S_{0} Q c_{s} \\
h(x) \equiv\left(1+x^{2}\right)^{\frac{1}{2}}-x
\end{gathered}
$$

The uniform monomer density of the lattice is $\rho_{0}$ and the Bjerrum length $Q=q^{2} / \varepsilon k_{B} T$ in terms of the elementary charge $q$ and the permittivity of the solvent $\varepsilon$. For a uniform hexagonal lattice, the area of the unit cell is $S_{0}=3^{\frac{1}{2}} H_{0}^{2} / 2$ where $H_{0}$ is the lattice spacing i.e. the distance between centeraxes of adjacent DNA helices $\left(\rho_{0}=1 / A S_{0}\right)$. Eq. (17) is, of course, an approximation though, on the whole, it does agree with recent careful measurements of the osmotic pressure of DNA suspensions by Raspaud et al [22] (to a good approximation, the pressure conforms to a universal form $\pi_{o s}=\rho_{0} k_{B} T \omega\left(c_{s} / \rho_{0}\right)$ independent of any model; the osmotic coefficient is also quite constant). At very high concentrations, the Oosawa approximation does seem to underestimate experimental pressures 23]. This has been attributed to a failure of the Oosawa model at close separations [24] but a calculation not invoking cylindrical symmetry still needs to be presented. Under conditions of tight packing, it is reasonable to neglect undulatory entropy. The bulk free energy is now the work of compressing the lattice from a state of infinite separation to the state at hand all the while keeping the chemical potential of the reservoir electrolyte fixed.

$$
\begin{gathered}
F_{\text {int }}\left[\rho_{0}\right]=L f_{\text {int }}+\text { const. } N=-3^{\frac{1}{2}} L \int_{\infty}^{H} d H^{\prime} H^{\prime} \pi_{o s}\left(H^{\prime}\right)+\text { const. } N \\
f_{\text {int }}=\left(\frac{k_{B} T}{2 Q}\right) I(w)
\end{gathered}
$$




$$
\begin{aligned}
I(w) & \equiv \int_{w}^{\infty} d y \frac{h(y)}{y} \\
& =-h(w)-\frac{1}{2} \ln \left[\frac{\left(1+w^{2}\right)^{\frac{1}{2}}-1}{\left(1+w^{2}\right)^{\frac{1}{2}}+1}\right]
\end{aligned}
$$

Hence, the chemical potential $\mu=\partial F_{\text {int }}\left[\rho_{0}\right] / \partial N$ of a DNA monomer at a fixed volume and buffer concentration may be expressed by

$$
\mu=\text { const. }+\frac{A k_{B} T}{4 Q} \ln \left[\frac{\left(1+w^{2}\right)^{\frac{1}{2}}+1}{\left(1+w^{2}\right)^{\frac{1}{2}}-1}\right]
$$

Note that the limiting forms of the logarithmic term are $-2 \ln w$ as $w \ll 1$ and $2 / w$ as $w \gg 1$; still, in practice, the variable $w$ is often of order unity.

We next apply eq.(12) to the deformed DNA lattice inside the phage whose density $\rho(\mathbf{r})=4 Q c_{s} / A w(\mathbf{r})$ at equilibrium is given by eq.([6) .

$$
\frac{\left(1+w^{2}(\mathbf{r})\right)^{\frac{1}{2}}+1}{\left(1+w^{2}(\mathbf{r})\right)^{\frac{1}{2}}-1}=k \exp \left(-\frac{2 P Q}{R_{c}^{2}(\mathbf{r})}\right) \equiv Z(\mathbf{r})
$$

where $k$ is a constant. This is rewritten as

$$
\rho(\mathbf{r})=\left(\frac{2 Q c_{s}}{A}\right) \frac{Z(\mathbf{r})-1}{Z^{\frac{1}{2}}(\mathbf{r})}
$$

implying that the dimensionless function $Z(\mathbf{r})$ must be larger than unity otherwise a solution to eq.(13) does not exist. Without knowing the precise structure of the DNA globule inside the phage, we already conclude from eq.(13) that tightly wound regions of curvature radius $R_{c} \simeq \sqrt{2 P Q} \simeq 8 \mathrm{~nm}$ must be depleted of DNA (persistence length $P=$ $50 \mathrm{~nm}$; Bjerrum length $Q=0.71 \mathrm{~nm})$. The tight bending of the DNA causes an increase in the curvature stress which has to be relieved by lowering the electrostatic stress (see fig. 1).

Let us now consider the profile about a cylindrical hole within a phage filled with DNA of genomic size. Then, the DNA almost fills the compartment of volume $V$ completely so we have from eq. (15)

$$
\frac{L}{A}=\int_{V} d \mathbf{r} \rho(\mathbf{r}) \simeq \frac{2 Q c_{s} V(k-1)}{A k^{\frac{1}{2}}}
$$

i.e.

$$
X \equiv \frac{L}{2 Q c_{s} V} \simeq \frac{k-1}{k^{\frac{1}{2}}}
$$

By way of example, let us reconsider the data on phage T7 of Cerritelli at al [5] which we previously analyzed in terms of a model of uniform density [2]. The $\mathrm{NaCl}$ concentration 
of the buffer was $0.1 \mathrm{M}$. The DNA genome of length $L$ is enclosed in a volume $V$ of about $77600 \mathrm{~nm}^{3}$ which almost equals the volume $V_{D N A}$ of the DNA lattice itself. Eq.(16) then yields $X=2.06$ and $k=6.08$. Accordingly, the radius $E$ of the (presumably single) hole free from DNA is computed from eq.(13):

$$
E^{2}=\frac{2 P Q}{\ln k}
$$

yielding $E \simeq 6 \mathrm{~nm}$. This should be compared with a hole radius of about $4 \mathrm{~nm}$ or possibly larger, based on the experimental spacing $(H=2.54 \mathrm{~nm})$ and estimates of the inner volume of bacteriophage $\mathrm{T} 7$ [2, 5]. An alternative route to a theoretical estimate for $E$ is via the (average) experimental spacing $\bar{H}$ : the volume $V_{D N A}$ of the DNA lattice is slightly less than $V$ and so $V \simeq L \bar{S}$. Hence, we have $X=2.10$ from eq.(16) and again $E \simeq 6 \mathrm{~nm}$. Similar conclusions apply to recent structural work on bacteriophage T4 [25]. If the phage is modeled as an ideal sphere of radius $R=41.5 \mathrm{~nm}$, the radius of the hole would be about $10 \mathrm{~nm}$ (setting $L=57800 \mathrm{~nm} ; c_{s} \simeq 0.3 \mathrm{M}$ ) from eq.(17). Olson et al [25] studied the DNA density which decreases inward starting from the outer capsid shell and there does appear to be a hole of radius $\sim 10 \mathrm{~nm}$. However, at present, it is difficult to make this comparison more quantitative. Finally, we can justify the neglect of density gradients and Coulomb integral terms in eq.(2); they are only of relative magnitude $H^{2} / R_{c}^{2}$ which is much smaller than unity.

We conclude from a simple model of close-packed DNA enclosed in phages, in which we relax the supposition of uniformity, that a hole (or holes) must occur free from DNA, at least at ionic strengths typical of laboratory or physiological conditions. The curvature stress in a tightly wound region of DNA becomes so high that the nonuniform Donnan effect can only compete up to a point; the DNA is thus forced out of such a region beyond a certain curvature given by eq.(17) (See fig. 2). It would be interesting to see if the DNA density may be approximated by eq. (13) - which is independent of the shape of the capsid (!) - but one has to be wary about the limitations of the present continuum analysis. At low densities, toward a hole, the DNA mesophase should become more disordered. Kasspidou and Van der Maarel have established that a hexagonal phase of short DNA rods melts into a cholesteric phase [9]. This happens, for instance, at an interaxial spacing of $H=3.7 \mathrm{~nm}$ in a $0.10 \mathrm{M} \mathrm{NaCl}$ solution. It is then predicted from eq.(14) that such a transition would occur at a radius of curvature $R_{c}=9 \mathrm{~nm}$ in the 
T7 phage [5], at least if we are allowed to extrapolate the results for short rods [9] to long DNA. At such nanoscales the concept of phases becomes very tentative of course. Anyway, the more disordered cholesteric "phase" surrounding the hole would still have an average spacing $H$ very similar to that of the coexisting hexagonal phase [9] in the bulk of the phage. Another possibility for disorder is an escape of a section of the DNA "into the third dimension" i.e. if a DNA strand aligns along the cylindrical axis of the hole. Such a state would seem to be unstable however.

The buffers used in [6, 25] also contain magnesium ions though not in excess. When genomic DNA is then packaged in bacteriophage T7, two Raman bands of the DNA are significantly perturbed with respect to DNA in solution [26]. Overman et al attribute this to enhanced magnesium concentrations within the phage [26. The effect of divalent ions has not been allowed for here although we note that magnesium is absent in the buffers used by Cerritelli et al [5].

Small angle X-ray scattering could be useful in trying to verify the profile predicted by eq. (14). However, it is well known that the scattering by phages is subtle with regard to interpretation [27]. The wavevector dependence of the scattering by lyotropic liquid crystals is peculiar in its dependence on the osmotic compressibility and the elastic moduli [28. It might be useful to extend theories pertaining to scattering from the bulk [15, 16, 18] to that from the DNA mesophase confined in phages. We remark that there is a length scale in the theory for a fluctuating splayless liquid crystal which turns out to be

identical to our decay length $\sqrt{2 P Q}$ in eq.(13) (in [15, 16, 18, there is also a competition of bending and osmotic stresses). Therefore, the DNA liquid crystal near the outer rim of the phage behaves much like a useful polymer liquid crystal but this is no longer true near the hole.

\section{Acknowledgment}

We thank Paul Jardine for many discussions on viruses and Rudi Podgornik for clarifying the status of the possible hexatic phase. One of us (F.S.) is grateful to the Dutch Foundation for Virological Research for a grant entitled "Virussen in katachtigen". 


\section{References}

[1] Hill, T. L.; Nanoletters, 2001, 1, 273

[2] Odijk, T.; Biophys. J., 1998, 75, 1223

[3] Ubbink, J.; Odijk, T.; Biophys J., 1995, 68, 54

[4] Ubbink, J.; Odijk, T.; Europhys. Lett., 1996, 33, 353

[5] Cerritelli, M.E.; Cheng N.; Rosenberg, A.H.; Mc. Pherson, C.E.; Booy, F.P.; Steven, A.C.; Cell, 1997, 91, 271

[6] Smith, D.E.; Tans, S.J.; Smith, S.B.; Grimes, S.; Anderson, D.L.; Bustamante, C.; Nature, 2001, 413, 748

[7] Kindt, J.; Tzlil, S.; Ben-Shaul, A.; Gelbart, W.M.; Proc. Natl. Acad. Sci. USA., 2001, 98, 13671

[8] Durand, D.; Doucet, J.; Livolant, F.; J. Phys. II France, 1992, 2, 1769

[9] Kassapidou, K.; van der Maarel, J.R.C.; Eur. Phys. J. B, 1998, 3, 471

[10] Strey, H.H.; Wang, J.; Podgornik, R.; Rupprecht, R.; Yu, L.; Parsegian, V.A.; Sirota, E.B.; Phys. Rev. Lett. , 2000, 84, 3105

[11] Grimes, S.; Jardine, P.J.; Anderson, D.; Adv. Vir. Res., 2002, 58, 255

[12] Jardin, P.J.; Anderson, D.; "DNA Packaging" in "The Bacteriophages", Ed. R.Calender, Oxford University Press, 2003, in press

[13] Arsuaga, J.; Vazquez, M.; Trigueros, S.; Sumners, D.W.; Roca, J.; Proc. Natl. Acad. Sci. U.S.A., 2002, 99, 5373

[14] Hud, N.V.; Downing, K.N.; Proc. Natl. Acad, Sci. U.S.A., 2001, 98, 14925

[15] Selinger, J.V.; Bruinsma, R.F.; Phys. Rev. A, 1991, 43, 2910

[16] Kamien, R.D.; Doussal, P.L.; Nelson, D.R.; Phys. Rev. A, 1992, 45, 8727

[17] De Gennes, P.G.; Phys. Lett., 1974, 47A, 123 
[18] De Gennes, P.G.; Mol. Cryst. Liq. Cryst., 1977, 34, 177

[19] Kleman, M.; J. Phys. France, 1980, 41, 737

[20] Oosawa, F.; Biopolymers, 1968, 6, 1633

[21] Oosawa, F.; Polyectrolytes. Marcel Dekker, New York, 1971

[22] Raspaud, E.; da Conceiao, M.; Livolant, F.; Phys. Rev. Lett., 2000, 84, 2533

[23] Strey, H.H.; Parsegian, V.A.; Podgornik, R.; Phys. Rev. E, 1999, 59, 999

[24] Hansen, P.L.; Podgornik, R.; Parsegian, V.A.; Phys. Rev. E, 2001, 64, 021907

[25] Olson, N.H.; Gingery, M.; Eiserling, F.A.; Baker, T.S.; Virology, 2001, 279, 385

[26] Overman, S.A.; Aubrey, K.L.; Reilly, K.E.; Osman, O.; Hayes, S.J.; Serwer, P.; Thomas, Jr, G.J.; Biospectroscopy, 1998, 4, S47

[27] Ronto, G.; Toth, K.; Feigin, L.A.; Svergun, D.I.; Dembo, A.T.; Comput. Math. Appl., 1998, 16, 617

[28] Ao, X.; Wen, X.; Meyer, R.B.; Physica A, 1991, 176, 63 


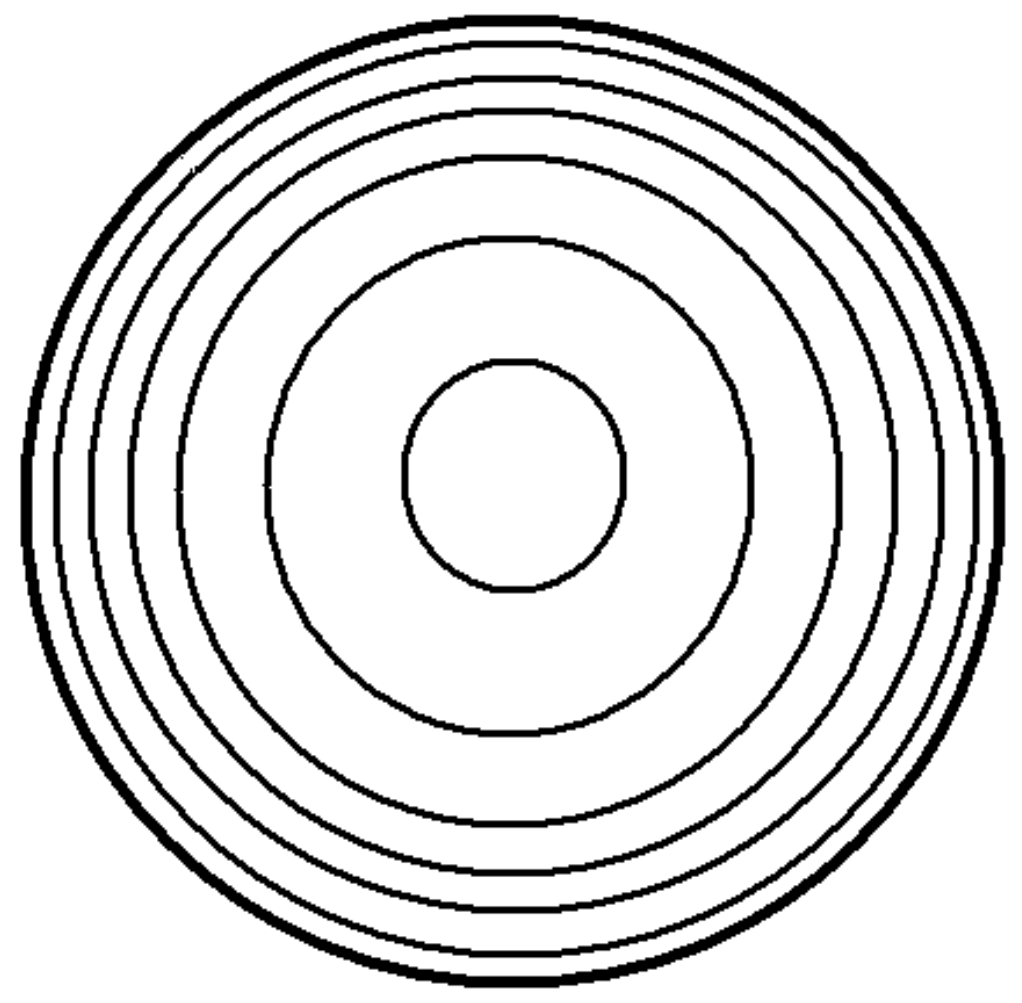

Figure 1: Schematic representation of DNA packed in a spherically shaped virus (plane perpendicular to the spool axis). The packing is locally hexagonal and becomes less close in the inner region of tight winding. 


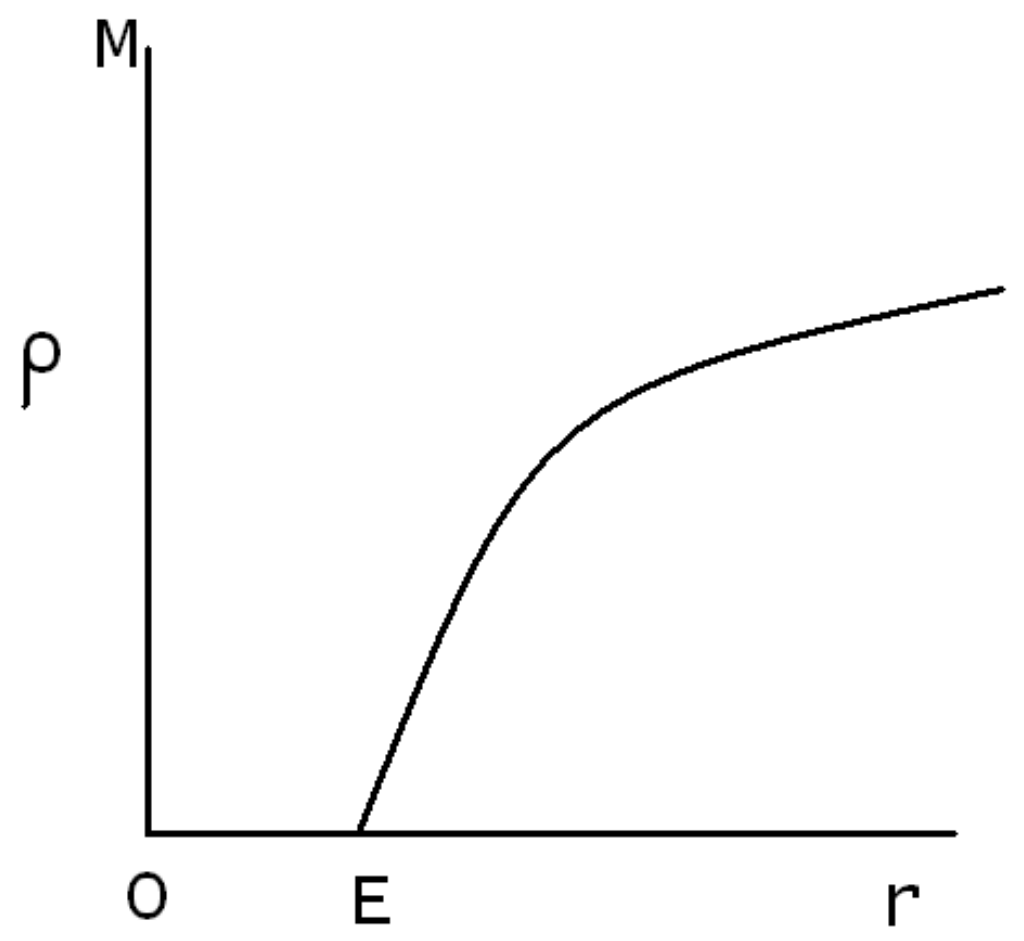

Figure 2: Coarse-grained DNA density in a plane through the central axis (MO) of a hole within a presumably spoollike region of DNA inside the phage. The size of the nonuniform region beyond $E$ is approximately equal to $\sqrt{2 P Q}$. 